\title{
Fingerprint Enhancement using Hierarchical Markov Random Fields
}

\author{
Rama Reddy K. N. V. and Anoop M. Namboodiri \\ IIIT Hyderabad \\ Gachibowli, Hyderabad 500 032, India \\ knvrreddyeresearch.iit.ac.in anoopeiit.ac.in
}

\begin{abstract}
We propose a novel approach to enhance the fingerprint image and extract features such as directional fields, minutiae and singular points reliably using a Hierarchical Markov Random Field Model. Unlike traditional fingerprint enhancement techniques, we use previously learned prior patterns from a set of clean fingerprints to restore a noisy one. We are able to recover the ridge and valley structure from degraded and noisy fingerprint images by formulating it as a hierarchical interconnected MRF that processes the information at multiple resolutions. The top layer incorporates the compatibility between an observed degraded fingerprint patch and prior training patterns in addition to ridge continuity across neighboring patches. A second layer accounts for spatial smoothness of the orientation field and its discontinuity at the singularities. Further layers could be used for incorporating higher level priors such as the class of the fingerprint. The strength of the proposed approach lies in its flexibility to model possible variations in fingerprint images as patches and from its ability to incorporate contextual information at various resolutions. Experimental results (both quantitative and qualitative) clearly demonstrate the effectiveness of this approach.
\end{abstract}

\section{Introduction}

Enhancement of fingerprint images is an essential step in any practical fingerprint based authentication system as fingerprint images that are acquired in real world are often noisy [17]. The noises and degradations arise due to poor skin conditions, varying finger pressure while acquisition, sensor noise and dry, wet or dirty fingers. Degradations in the quality of the acquired image results in spurious minutiae points or removal of real ones, thus directly affecting the ability of algorithms to reliably match the fingerprints.

Approaches to enhancement of fingerprints can be divided into two primary groups. Methods that model the noise to remove it includes techniques such as median fil-

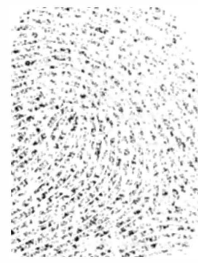

Input

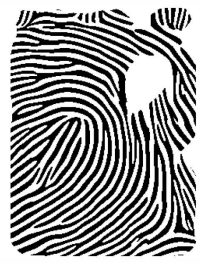

HMRF

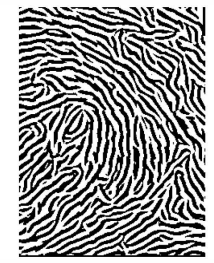

Gabor

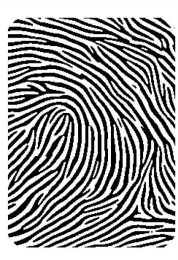

Smoothed
Figure 1. Enhancement result using the proposed method as well as multi-stage Gabor filtering and its smoothed version.

tering, Wiener filtering [9]), histogram equalization, contrast enhancement. Such techniques are effective only with a limited set of noises and usually serve as preprocessing steps for further levels of enhancement. Popular approaches model the fingerprint structure, and depending on the suitability of the model to the observed fingerprint, the output quality varies considerably. Figure 1 shows the result of enhancement of a degraded fingerprint using a multi-stage Gabor filter based enhancement and its smoothened version as compared to the proposed approach in this work (HMRF). The ability of a technique to recover information from a degraded fingerprint depends on the suitability of the assumptions in the model as well as the ability of the technique to incorporate contextual information.

Majority of the techniques that are currently employed in fingerprint image enhancement involve contextual filters. The parameters that guide these filters include local ridge orientation, frequency, reliability, etc. Early attempts included the use of elongated filters applied along the ridge direction [18]. Approaches that carry out the filtering completely in the frequency domain [19] or a mixture of spatial and frequency domain such as Short time Fourier Transform (STFT) [6] have been attempted.

The most popular method of spatial domain filtering uses a bank of Gabor filters as proposed by Hong et al. [12]. Gabor filters have the optimal joint resolution in both spatial and frequency domains. Further attempts for modifications and extensions to adaptively employ this method were made by Greenberg et al. [9], Bernard et al. [2], and Yang et 
al. [21]. The model assumes the signal at any local region to be a set of equidistant parallel ridges or a sinusoidal pattern with a single direction and frequency. This assumption is violated at high curvature regions such as singularities, and places where ridge widths and distances vary. Further, the accuracy of the filters dips where contextual information such as ridge frequency and local orientation cannot be reliably obtained such as regions corrupted by creases and smudges. Filters that are controlled by parameters can be either isotropic or anisotropic [16], where isotropic filters preserve the information while fail to recover many regions and anisotropic introduce many spurious structures. Hence, there is always a trade off involving level of enhancement and the generation of spurious fingerprint features.

Our approach is motivated by two observations: i) The above tradeoff between enhancement and spurious structures can be improved with a tight model for fingerprints. i.e., the underlying model should allow all fingerprint structures while suppressing as much noise as possible. ii) Strong use of context to narrow down the model in degraded regions can help in recovery of highly degraded regions with minimal information. To achieve the first goal, we model the fingerprint using a set of image patches learned from clean fingerprint images. To incorporate the contextual information, we model the enhancement process as that of inferring the most likely set of patches that generated the observed image. The contextual information is incorporated using constraints that are enforced between neighboring patches. We note that the resulting inference can be readily modeled using a Markov Random Field, where the observations are image patches. To incorporate contextual constraints, we extend the model to a hierarchical version, where each layer of the model works on different constraints such as orientation field, ridge continuity, etc.

In the past, Bayesian and MRF models were formulated to reliably extract the orientation fields from noisy fingerprints. Dass et al. [7] use quality and smoothness pairs as priors to extract orientation field. This method also iteratively modifies the orientation field around the singularities to obtain a more robust estimate of orientation field. However, this approach cannot recover orientation field from large patches of poor quality. Lee and Prabhakar [15] address this issue by using training samples of each orientation as priors. However, Orientation field alone cannot entirely enhance the fingerprint image especially the regions with heavy smudging where most of the information is lost.

In this work, we present a unified approach that performs fingerprint enhancement, orientation field extraction, classification, minutiae detection and ridge frequency estimation, using a multi-layer MRF model. Hierarchical MRF models are known to handle region geometry and spatial interaction effectively [10]. Working with different patch-sizes on two layers aids in better restoration of the noisy regions using

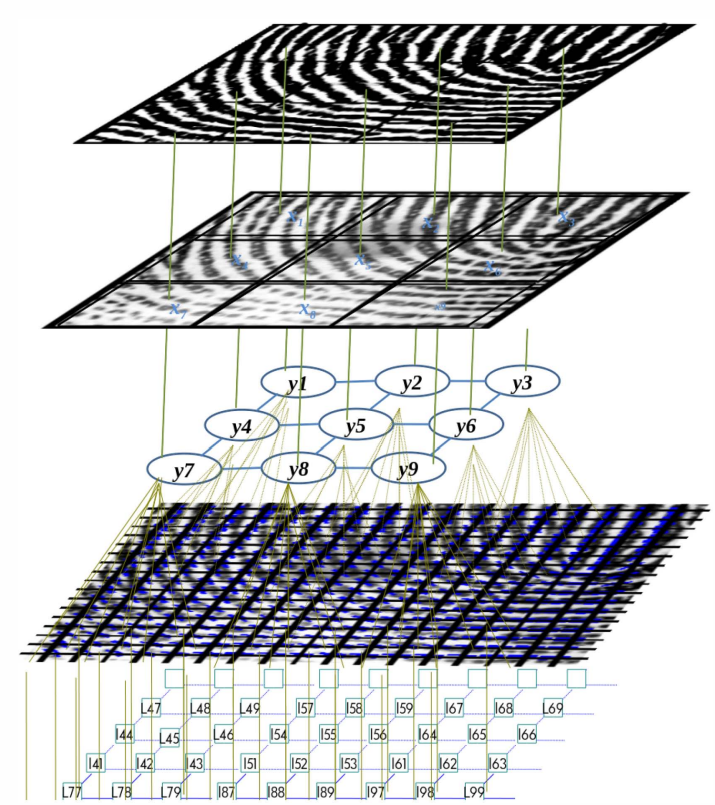

Figure 2. Two-stage MRF model with Ridge patch layer $\left(X_{i}\right)$ and Orientation layer below. Restored pathes are shown at the top.

whatever good quality regions are present in the fingerprint.

\section{Overview of the Process}

Consider a degraded fingerprint image that is divided into a set of square patches, $X_{i}$. We model the restoration process as that of estimating the most likely set of ideal patches, $Y_{i}$, that could generate $X_{i}$, along with satisfying the spatial relationship amongst them. The top layer of the MRF model concentrates on this task as shown in Figure 2. Note that our observation patches, $X_{i}$, are overlapping to ensure the spatial continuity of ridges in the restored image. However, in highly degraded images, the image potentials obtained from the patches are often ambiguous to carry out the estimation. We employ a second layer of smaller orientation patches to guide the selection of ridge image patches. Our restoration is an iterative process that oscillates between two layers.

In Figure 2, the ideal (restored) patch $Y_{5}$ depends not only on the observed patch $X_{5}$, but also on the context of its neighbors, $Y_{2}, Y_{4}, Y_{6}$ and $Y_{8}$. For example, the number of ridges at the left edge of $Y_{5}$ should be equal to the number of ridges at the right edge of $Y_{4}$ and at the same spatial locations. As mentioned before, the goal of restoration at ridge level is estimating a set of ideal patches, denoted as $\chi$, that are possible ridge patterns in a fingerprint, which also satisfies the spatial association, $p\left(Y_{i}, Y_{j}\right)$ at neighboring patches. Let the orientation information of these ideal patches be labeled as $O\left(Y_{i}\right)$. Note that all the orientations stay with in the range 0 to $\pi$. The notations introduced in this section are followed in the rest of the paper. 
We model the Orientation field smoothness using a class of spatially smooth priors as described in [7] in the bottom layer. Though this model alone does not provide a robust orientation estimate at higher noise levels, iteratively updating the orientation field using the information from ridge layer restoration along with the smooth priors gives a good estimate of orientation field. In the orientation layer, the principal gradient $l_{u}$ of a block $B_{u}$ not only depends on the gradients with in the block but also on the neighborhood principal gradients, $\left(l_{v}\right)$. We model this dependence as a $3 \times 3$ neighborhood $\left(N_{u}\right)$. The block-wise orientation field, $\mathrm{O}\left(B_{u}\right)$ for this layer is initialized using method presented in [1].

Along with the spatial interaction within the layers, we also establish the inter-layer connectivity information. The orientation information from the bottom layer blocks, $\mathrm{O}\left(B_{u}\right)$, is used to adjust the ridge patches in the top layer, while the orientation of bottom layer is updated using the orientation of the ridge patches, $O\left(Y_{i}\right)$. For each node $i$ in the ridge layer (top layer), the corresponding node neighborhood at the orientation layer (bottom layer) is denoted as $N Y_{i u}$. Let $O^{n}()$ denote the current orientation state of any layer after 'n' iterations. Let $P Y_{i u}$ denote the portion of ridge patch $Y_{i}$ corresponding to the block $B_{u}$ in orientation layer. The neighborhood takes a pyramid structure with each patch in top-layer (ridge restoration layer) corresponding to multiple patches ( $3 \times 3$ in our case) in bottom layer as shown in figure 2 .

\section{Hierarchical MRF Framework}

The restoration model involves a set of ideal training patches, $\chi$, and the potential functions for the multi-layer MRF-structure. The method of storing the patches is also critical for computational efficiency.

\subsection{Ideal patch extraction for restoration layer}

This stage involves the extraction of ideal prior patterns that are consistent and model the variations in the ridge structures of the fingerprints. To achieve this, we efficiently discretize the possible finger print patterns to sample all the minutiae patterns, ridge densities, singularity patterns, etc. Henry [11] has proposed a fingerprint classification scheme that divides fingerprints into five common classes, namely left loop, right loop, whorl, arch and tented arch. A few variations from these such as double loop have also been noted. These classes partition all types of singularities and orientation field variations. Therefore, we chose a set of $\mathrm{N}$ high quality fingerprints from each of the different classes as training samples. The value of ' $N$ ' should be chosen such that it effectively covers the minutiae patterns at various ridge densities. For a given patch-size 'P', we extract a patch from test fingerprints with an overlap of 'P/2' with its neighboring patches $(2 P \times 2 P)$. Bigger patches are chosen

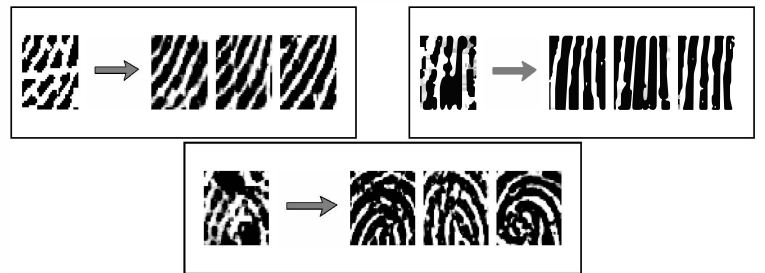

Figure 3. Input fingerprint patches and matching ideal ones at various resolutions $(30 \times 30,40 \times 40$ and $50 \times 50$ in clockwise order $)$.

to avoid the loss of pattern information at the edges when the test patches are registered to the observations, $X_{i}$. After patch extraction, all the patches are normalized to zero mean and unit variance and are stored in the ideal patch database along with their orientation field $\mathrm{O}\left(Y_{j}\right)$, ridge frequency $\mathrm{F}\left(Y_{j}\right)$ and filter-enhanced forms $\mathrm{E}\left(Y_{j}\right)$ and minutiae/singularity information.

As we can see from the figure 3 , large patch sizes should be avoided since they become pattern specific.

\subsection{Potential functions and Priors}

During the learning phase the potential functions are learnt from the training data. The inference phase involves computing the marginals of posterior distribution $\mathrm{p}\left(Y_{i} \mid X\right)$, for all nodes $\mathrm{i} \in[1, . . \mathrm{N}]$ and computing the maximum likelihood estimate (MLE) of the priors for all the blocks, $B_{u}$.

Ridge patch association potential: We model the association potential, $\phi\left(Y_{i}, X_{i}\right)$ as function that gives the minimum squared distance between the ideal patches $Y_{i}$ and the observation patch $X_{i}$. We register the patches $Y_{i}$, which are larger, with $X_{i}$ using the orientation field and its reliability. We find the rotation angle, $\widehat{\Theta}$, and translation, $\widehat{T}=\left[t_{x}, t_{y}\right]$ that minimizes the distance between $Y_{i}$ and $X_{i}, \mathrm{~d}\left(\mathrm{R}\left(Y_{i}, \widehat{\Theta}, \widehat{T}\right), X_{i}\right)$, which is the normalized sum of squared differences between the transformed patch candidates $\mathrm{R}\left(Y_{i}, \widehat{\Theta}, \widehat{T}, X_{i}\right)$ and $X_{i}$. The potential between degraded fingerprint patch $X_{i}$ and candidate patch $Y_{i}$ is given by:

$$
\phi\left(Y_{i}, X_{i}\right)=\exp \left(-\frac{\left.d\left(R\left(Y_{i}, \widehat{\Theta}, \widehat{T}\right), X_{i}\right)\right)}{2 \sigma_{n}^{2}}\right),
$$

where $\sigma_{n}$ is a noise parameter and can be modeled through leave one out cross-validation technique [20].

Ridge patch Interaction potential: The Ridge patch Interaction potential, can be modeled as the normalized sum of squared differences between the patch candidates $Y_{i}$ and $Y_{j}$ in the overlap region at their common edge at the neighboring nodes $\mathrm{i}$ and $\mathrm{j}$. The Ridge patch Interaction potential between candidates $Y_{i}$ and $Y_{j}$ at nodes $\mathrm{i}$ and $\mathrm{j}$ is given by:

$$
\psi\left(Y_{i}, Y_{j}\right)=\exp \left(-\frac{d\left(Y_{i}, Y_{j}\right)}{2 \sigma_{c}^{2}}\right),
$$

where $\sigma_{c}$ is noise parameter, modeled as before [20]. 
Quality Prior : Let $v_{P}$ be the normalized gradient vector, $\left(\cos \left(\theta_{P}\right), \sin \left(\theta_{P}\right)\right)$ at a pixel $P=(x, y) \in$ block $\left(B_{u}\right)$. Then the quality prior $\left(Q_{p}(u)\right)$ indicates how uniformly the gradients $\left(v_{P}\right)$ are aligned with in $B_{u}$.

$$
Q_{p}(u)=\sum_{P \in B_{u}} v_{P} v_{P}^{T}
$$

The block-wise Maximum Likelihood Estimate (MLE) of block principal gradient $\left(l_{u}\right)$ is given by the eigen vector corresponding to the maximum eigen value of $Q_{p}(u)$. The reliability $\left(W_{u}\right)$ of $\operatorname{block}\left(B_{u}\right)$ is given by

$$
W_{u}=\frac{\lambda_{1}-\lambda_{2}}{\lambda_{1}+\lambda_{2}}
$$

where $0 \leq W_{u} \leq 1$ with $W_{u}=1$ indicating that all the gradient vectors within the block point in the same direction and hence, the block information is reliable and should be favored more in the likelihood estimate. $\lambda_{1}$ and $\lambda_{2}$ are the maximum and minimum eigen values of the quality prior $Q_{p}(u)$.

Spatial smooth priors : The spatial dependence of a block, $B_{u}$ on principal gradient vectors of neighboring blocks, $N_{u}$ is incorporated through these priors, $N_{p}(u)$. If $W_{v}$ represents the reliability of each of these neighboring blocks and $l_{v}$ represents the principal gradient of one of the neighborhood blocks $\left(N_{u}\right)$ of block, $B_{u}$, then the spatial smooth prior $N_{p}(u)$ is given by

$$
N_{p}(u)=\frac{1}{W_{T}} \sum_{v \in N_{u}} W_{v} l_{v} l_{v}^{T},
$$

where $W_{T}=\sum_{v \in N_{u}} W_{v}$ is normalization constant equal to the sum of the neighborhood radiabilities. The unit eigen vector of this weighted matrix $N_{p}(u)$ corresponding to maximum eigen value gives the principal direction of the neighborhood $N_{u}$ of block $B_{u}$.

Ridge patch Orientation potential : The Ridge patch Orientation potential is split into a conditional term $\rho\left(Y_{i} \mid B_{u}\right)$ and a marginal term $\zeta\left(B_{u}\right)$. The conditional term is modeled as the normalized sum of squared differences between the orientations, $\mathrm{O}\left(Y_{i}\right)$ and $\mathrm{O}\left(B_{u}\right)$ and the marginal term indicates the reliability, $W_{u}$ of the corresponding block $B_{u}$.

$$
\rho\left(Y_{i}, B_{u}\right)=\exp \left\{-\sum_{B_{u}} W_{u} d\left(O\left(Y_{i}\right), O\left(B_{u}\right)\right) /\left(2 \sigma_{o}^{2}\right)\right\},
$$

where $\sigma_{o}$ is modeled through cross validation [20].

Inter-layer Orientation prior : The dependence of a block, $B_{u}$, on the ridge orientations of the top layer, $\mathrm{O}\left(Y_{i}\right)$ is modeled through these priors, $f_{p}(u)$. If $l_{f}$ gives the principal gradient vector of block, $\mathrm{O}\left(P Y_{i u}\right)$, prior is given by

$$
f_{p}(u)=l_{f}
$$

\section{Multi-grid MRF Inferences and messages}

To compute the Maximum Posterior Marginal (MPM) estimate and obtain the ideal patch for node $\mathrm{i}$ in the ridge layer, we use belief propagation inference to solve the MRF [5]. Specifically, we use loopy belief propagation (LBP) [8] as our graph contains cycles and LBP has been empirically shown to perform well for several hard problems.

In the bottom layer, we perform iterative orientation field estimation using quality and smoothness priors as well as information from ridge enhancement layer. We choose ICM as inference to solve the MRF at this layer, since it is computationally faster and also suits the basic goal of our restoration. This inference algorithm ensures spatial smoothness in the orientation field and also reliably updates the ridge patches in top layer from reliable orientation patches. There are four different types of messages, namely the message passed between neighboring patches $\left(Y_{i}, Y_{j}\right)$ in the ridge layer, the spatial smoothness priors $\left(l_{u}, l_{v}\right)$ in the orientation field restoration layer, and the messages to hierarchically corresponding patch neighborhoods, $N Y_{i u}$ and $P Y_{i u}$ in the other layers. In the following segments, $\alpha$ denotes the normalization factor for these messages.

- Intra - Ridge layer Restoration messages : The message propagated from node $i$ to node $j$ in the ridge layer during the nth iteration is given by :

$$
\begin{array}{r}
m_{i, j}^{n}\left(Y_{j}\right)=\alpha \int \psi\left(Y_{i}, Y_{j}\right) \phi\left(Y_{i}, X_{i}\right) \\
\prod_{k \in N_{\boldsymbol{R}}, k \neq j} m_{k, i}^{n-1}\left(Y_{i}\right) \prod_{h \in N Y_{i u}} m_{h, i}^{n-1}\left(Y_{i}\right) d Y_{i}
\end{array}
$$

- Orientation - Ridge layer messages : The message propagated from a node $u$ in the orientation layer to a node $\mathrm{i}$ in the ridge layer during the nth iteration represented as is given by :

$$
m_{i, r}^{n}\left(Y_{j}\right)=\alpha \int \rho\left(Y_{i}, B_{u}\right) \prod_{q \in N Y_{i u}} m_{q, i}^{n-1}\left(Y_{i}\right) d Y_{i}
$$

\subsection{Belief propagation}

Let $\mathrm{P}(\bar{Y}, \bar{X})=\mathrm{P}\left(Y_{1}, \ldots, Y_{N}, X_{1}, \ldots, X_{N}\right)$ be the joint probability of observing $X_{1}, \ldots, X_{N}$ when the corresponding underlying labels are $Y_{1}, \ldots . . Y_{N}$. The ridge interaction potential $\psi\left(Y_{i}, Y_{j}\right)$ denotes the pairwise compatibility between two neighboring labels, $Y_{i}$ and $Y_{j}$ and $\phi\left(Y_{k} \mid X_{k}\right)$, the likelihood that the label $Y_{k}$ generates the observed patch, $X_{k}$. The joint probability can now be formulated as :

$$
\begin{aligned}
P(\bar{Y}, \bar{X}) & =P\left(Y_{1}, \ldots, Y_{N}, X_{1}, \ldots, X_{N}\right) \\
& =\prod_{(i, j)} \psi\left(Y_{i}, Y_{j}\right) \prod_{k} \phi\left(X_{k} \mid Y_{k}\right),
\end{aligned}
$$


The first product is over all the neighboring pairs of nodes,i and $\mathrm{j}$. The belief propagation algorithm updates messages, $m_{i j}$, from node $\mathrm{i}$ to node $\mathrm{j}$, which are used to infer the state at node $\mathrm{j}$. The state of node is based on the messages it receives and the process is repeated till convergence. The initial messages, $m_{i j}$ s are set to column vectors of 1's of dimensionality of the variable $Y_{j}$. Similarly, for the messages coming from the Orientation layer.

However, in a generic MRF framework $\psi\left(Y_{i}, Y_{j}\right)$ and $\phi\left(Y_{k}, X_{k}\right)$ have huge number of components. It becomes computationally infeasible to compute $m_{i j}^{n}\left(Y_{j}\right)$. Hence, we need a technique to prune components to be computationally reasonable and meaningful. We use the information from the ridge patch orientation potential $\rho\left(Y_{i}, B_{u}\right)$ to prune the components and learn from a subset sampled from training set.

The restored ridge image is obtained by estimating the Maximum Posterior Marginal (MPM) $\operatorname{argmax}_{Y_{i}} p\left(Y_{i} \mid X\right)$ at all nodes $i \in[1, \ldots N]$ in ridge layer

$$
\begin{array}{r}
p\left(Y_{i} \mid X\right) \approx \alpha \phi\left(Y_{i}, X_{i}\right) \prod_{k \in N_{\boldsymbol{R}}, k \neq i} m_{k, i}^{n-1}\left(Y_{i}\right) \\
\prod_{r \in N Y_{i u}} m_{r, i}^{n-1}\left(Y_{i}\right),
\end{array}
$$

\subsection{Iterative Conditional Modes}

ICM is an iterative procedure that maximizes the conditional distribution of block principal gradient, $l_{u}$ given its neighbors and other priors. Each update of ICM algorithm increases the value of posterior distribution given below

$$
\begin{array}{r}
\pi\left(l \mid Q_{p}(u), N_{p}(u), f_{p}(u)\right) \propto \exp \left\{\tau^{2} W_{u}\left(l_{u}^{T} Q_{p}(u) l_{u}\right)\right. \\
\left.+\lambda \Sigma_{v \in N_{u}}\left(l_{u}^{T} N_{p}(u) l_{u}\right)+\gamma\left(l_{u}^{T} f_{p}(u) l_{u}\right)\right\}
\end{array}
$$

Hence the estimate of $l_{u}$ after each iteration can be obtained by the eigen unit vector corresponding to the maximum eigen value of the weighted matrix

$$
\begin{array}{r}
\frac{\tau^{2} W_{u}}{\tau^{2} W_{u}+\lambda+\gamma} Q_{p}(u)+\frac{\lambda}{\tau^{2} W_{u}+\lambda+\gamma} N_{p}(u) \\
+\frac{\gamma}{\tau^{2} W_{u}+\lambda+\gamma}\left(f_{p}(u)\right)
\end{array}
$$

The parameters $\tau^{2}$ represents the ratio of contribution of each block towards its directional field, $\lambda$ represents the ratio of contribution of block's neighbors towards its directional field and $\gamma$ represents the ratio of contribution of ideal patches $\left(Y_{i}\right)$ towards the orientation field.

\subsection{Multilayer evaluation}

- Intra - Ridge layer restoration messages are updated alternatively as disjoint graphs, instead of updating each node in every iteration for computational efficiency.
- Ridge layer to Orientation layer message is approximately evaluated as the MPM estimate of $Y_{j}$ as shown in equation 10 to avoid computational difficulties involved in computing the exact integral.

- The messages within the ridge layer are pruned based on the weight of the components and at every iteration the MAP estimate of ridge layer is used to update the orientation field in the bottom layer.

One of the other advantages of our formulation is, from multiple low-resolution patches we can directly estimate the ideal patches at high resolution, thus combining fingerprint enhancement and resolution improvement into one process.

\section{Experimental Results and Discussions}

We split the fingerprint image into overlapping patches, $X_{i}$, for enhancement and each of these patches is locally normalized through adaptive information from smaller patches. Reliability and mean from smaller level patches are used to set mean for larger patches to normalize locally. Adaptive normalization must be performed in-order to remove the background from the image patches and ensure that ridges of the training patches are at the same intensity as image patches and to ensure that the noise from global level does not effect the local patches. For a typical 500 dpi image we choose a patch size of $30 \times 30$ and hence, ideal patches have a size of $60 \times 60$. An overlap of 5 pixels is allowed between the neighboring patches.

The orientation blocks are chosen to be of size $10 \times 10$. Hence a single patch in ridge layer corresponds to 9 blocks in orientation layer. Larger patch size in ridge layer indicates that prior is defined on a large neighborhood making it more powerful. Patches, $Y_{i}$ with low confidence scores are masked to avoid spurious minutiae in the restored fingerprints. This is because low MPM value indicates the non-reliability of neighbors and prior information is used to restore the corresponding patch.

\subsection{Qualitative Analysis}

We first focus on the visual analysis and comparison of state of art technique and the proposed HMRF based technique. For comparison, we use the best implementation we could obtain of a Gabor filter-based enhancement technique. It uses a two-stage enhancement where orientation estimates obtained after the first stage is used for filter the original image once again [4]. Noisy fingerprints were chosen from FVC 2006 [13] database and results of both the techniques have been qualitatively compared by a human examiner. Figure 4 provides a qualitative comparison on some of the fingerprints. Binarized and masked finger prints using the proposed method are shown in the second column. The results of Gabor based enhancement techniques [12], 
with parameters applied as described in the [4] are shown in third column. The fourth column shows the result of Gabor filtering, masked using a orientation coherence based reliability threshold.

In the first row of Figure 4, large parts of ridge information is lost due to poor contact of a dry fingerprint. Our algorithm is able to judge most of the lost patterns from the neighborhood patterns in this case. Similarly, in figure 4, row 2 , due to wet contact heavy smudges are produced in the fingerprint. Our algorithm could restore some part of it reliably. Some information could not be restored since prior information and large parts of neighborhood information were not reliable enough to restore those patches. In figure 4 , row 3 , though significant information is lost in a part of the fingerprint, it has been reliably restored from the neighboring ridge information. In the last row of figure 4, heavy creases cause degradations in the Gabor result resulting in spurious structures. Since ideal patches contain no creases our restoration process reliably eliminates these creases.

Figure 5 shows the result of minutiae extraction from the enhanced results using a state-of-the-art commercial fingerprint matching software. We can observe the missing minutiae and singularities extracted through our approach. These results indicate the robustness of our approach to higher noise, at various levels.

Careful analysis of the results show that the minutiae types are often of flipped or its location moves from one ridge to its neighbor. This is an artifact of the set of patches that are used in training and could be improved by using a larger set of patches. Moreover these changes does not affect the matching process as seen later as the minutiae patterns remain the same.

\subsection{Quantitative Analysis}

Quantitative Analysis was done using an experiment that compares the minutiae extracted through different techniques. To obtain a controlled dataset with known ground truth, 24 fingerprints were generated using SFinGe [3] software. The generated images can be categorized into three noise levels: medium, high and very high. SFinGe software provides an option of adding various noises types to the fingerprint such as improper contact (dry and wet), scratches, background/sensor noise, creases etc. Medium to high levels of noise was added to the generated fingerprints and compared with the ground truth of these noisy fingerprints provided by SFinGe.

To obtain a better understanding of the power of the proposed method, we used two different variants of the Gabor filter based enhancement method as described below:

- FAGE - Fully Automated Gabor Enhancement: This is the regular two-stage Gabor filter method as

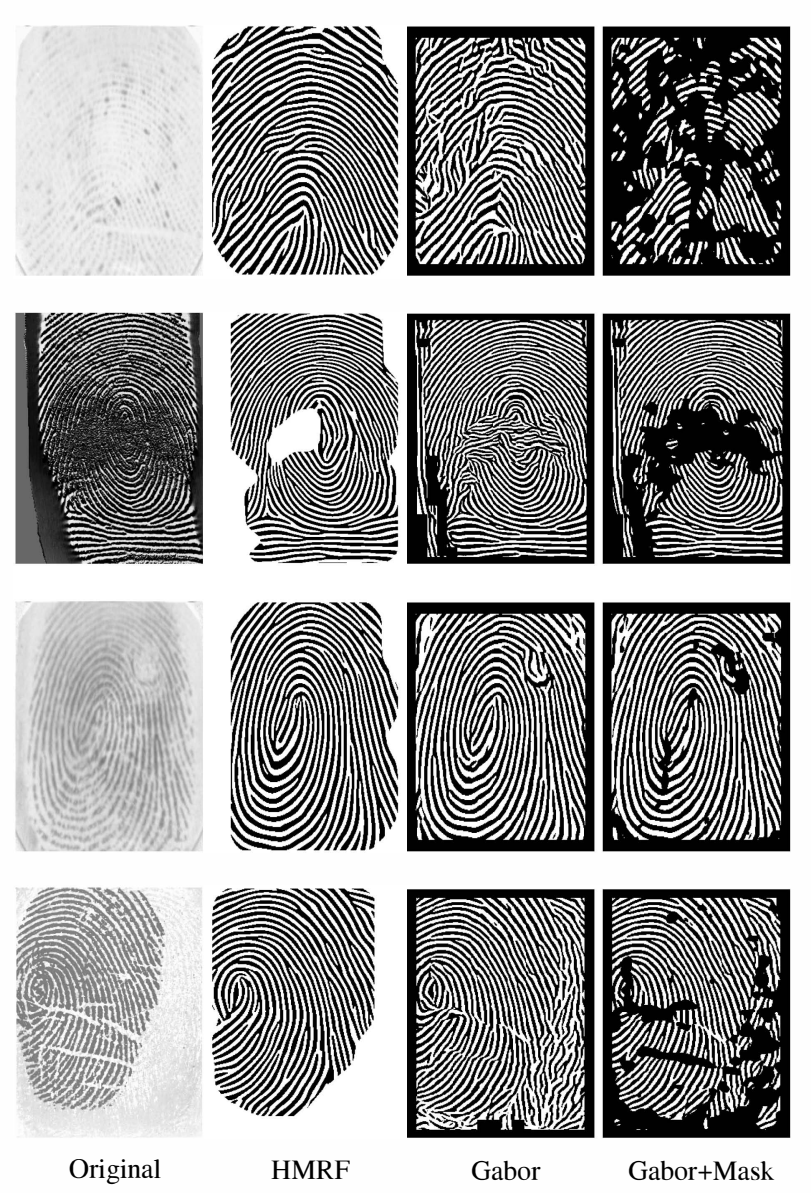

Figure 4. Restoration of fingerprints with various degradations using the proposed and Gabor [4] methods.

mentioned in previous experiments [4]. The Gabor filter is applied twice sequentially and fingerprint segmentation is done based on variance for noise suppression and image enhancement.

- SAGE - Semi Automated Gabor Enhancement: This is a human assisted version of Gabor filter, where a human expert provides inputs where reliable estimates of local Orientation $(\mathrm{O})$ and Frequency(f) are not available [4]. In fact, we provide the ideal orientation and local frequency for the synthetic fingerprints. The reader might note that such a method in not practical in real world as ideal values of orientation and frequency are never known for noisy images. However, we can use the results as the limit that any automatic enhancement algorithm can try to achieve.

Minutiae extraction is done for the enhanced image using a state-of-the-art commercial software and compared to the ground truth minutiae. Minutiae are given a 10-pixel tolerance range and optimal pairing is determined as given in [14]. The three techniques are compared through the fol- 

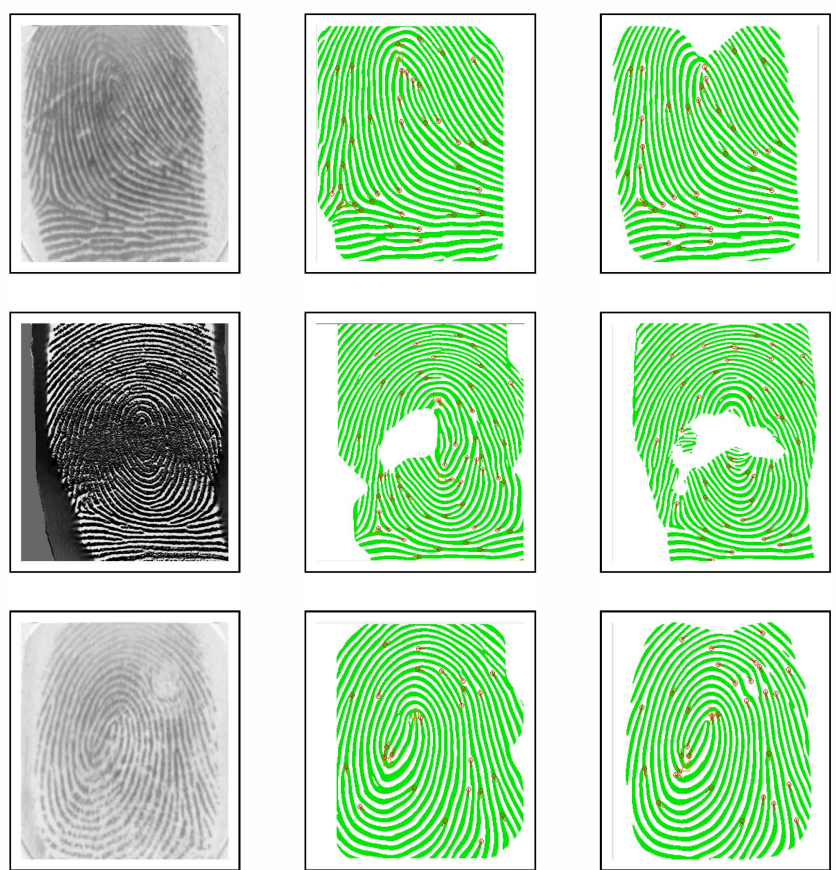

Original

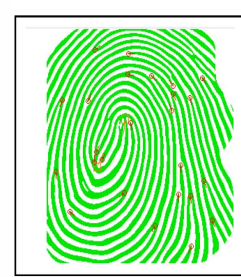

HMRF

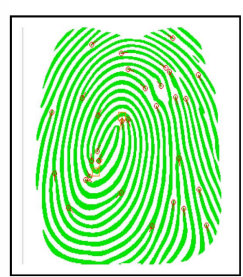

Gabor
Figure 5. Minutiae extraction results from images enhanced using proposed and Gabor [4] methods.

\begin{tabular}{|l|c|c|c|c|}
\hline FAGE & HMRF & SAGE & GT & Minutiae Count \\
\hline \hline 1045 & 898 & 909 & 910 & Detected \\
\hline \hline 294 & 184 & 132 & N/A & Missed \\
\hline \hline 429 & 172 & 131 & N/A & Spurious \\
\hline \hline $32.3 \%$ & $20.2 \%$ & $14.5 \%$ & N/A & Missed (\%) \\
\hline \hline $47.1 \%$ & $18.9 \%$ & $14.4 \%$ & N/A & Spurious (\%) \\
\hline
\end{tabular}

Table 1. Results of minutiae extracted from Fully Automated Gabor Enhancement (FAGE), Proposed Method (HMRF), and Semi Automated Gabor Enhancement (SAGE).

lowing metrics:

- Missed minutiae: Count of minutiae in ground-truth that have no pairing with the extracted minutiae of enhanced image.

- Spurious minutiae: Count of extracted minutiae in enhanced image that have no pairing with the minutiae in ground-truth.

Table 1 summarizes the results of the experiment. Our technique reports results with accuracy close to SAGE and a significant improvement over the fully automated techniques with the Missed minutiae count coming down by 12 percent and spurious minutiae count more than halved.

Figure 6 provides a visual illustration of some of the images from the above experiment. One image from each

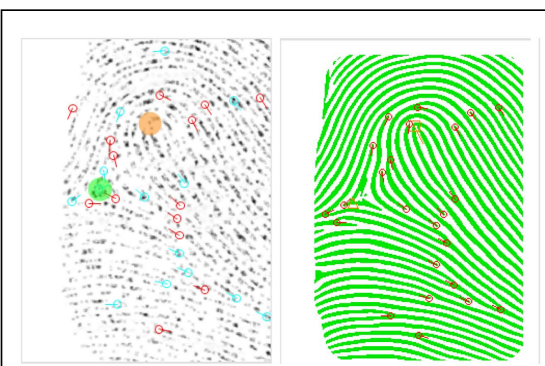

(a) Ground Truth(28)

(b) FAGF $(24,7,3)$

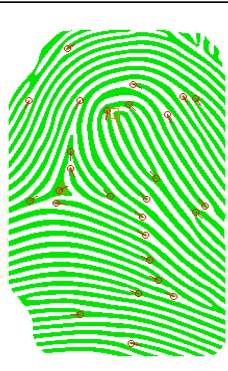

(c) $\operatorname{HMRF}(27,4,3)$

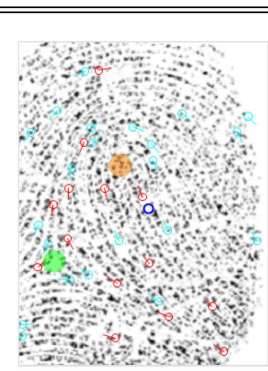

(d) Ground Truth(36)

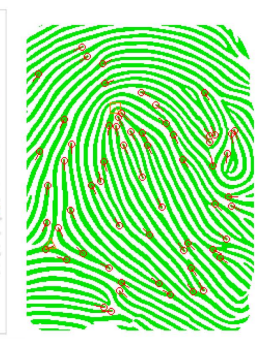

(e) $\operatorname{FAGF}(59,12,35)$

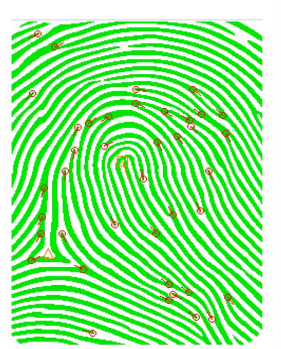

(f) $\operatorname{HMRF}(42,10,16)$

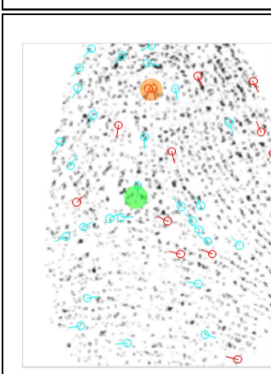

(g) Ground Truth(39)

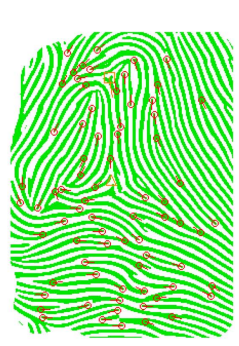

(h) FAGF $(67,36,64)$

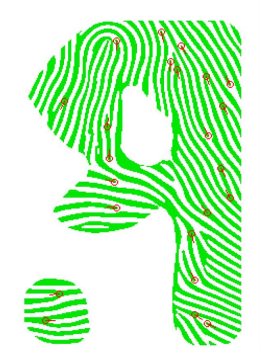

(i) $\operatorname{HMRF}(24,30,15)$
Figure 6. Minuitae maps with (DM,MM,SM) information for medium, high and coarse noised fingerprints along with Groundtruth.

of the three categories of fingerprints namely medium, high and very high noise are selected. Stated below the images are the counts of detected minutiae(DM), missed minutiae(MM) and spurious minutiae(SM). We can observe that the spurious minutiae are significantly reduced by our method. This is because the restoration is done based on training patterns that do not give rise to many spurious structures. Figure 8 gives an example of a latent fingerprint that is restored using the proposed approach.

Other potential applications that are enabled by our restoration procedure include determination of orientation field and type of the fingerprint. After evaluating the ideal restoration $Y_{j}$, for an observed patch $X_{i}$ in ridge layer finding the orientation field involves simply substituting the orientation field of the restored patch in the position of the observed patch. If we previously extract the singularity information in the training fingerprints, then finding the singularity in the restored image is simply locating the restored 

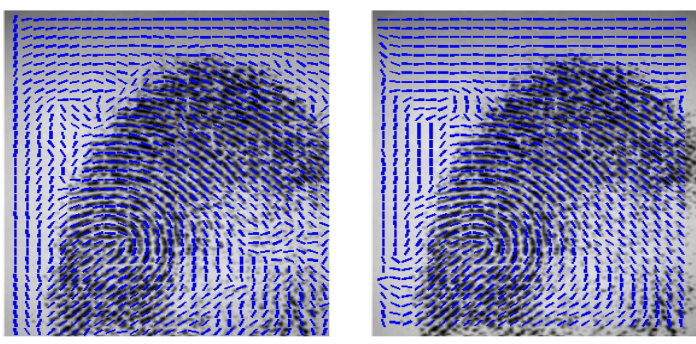

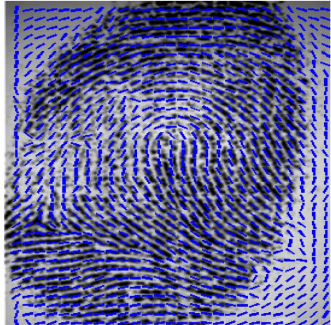

Gradient

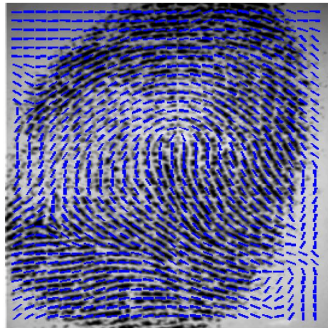

HMRF
Figure 7. Orientation fields extracted traditional gradient based and the proposed methods on two images.

patch with singularity. Extracted directional fields of some examples are shown in Figure 7.

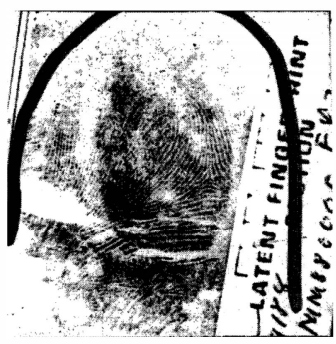

Latent

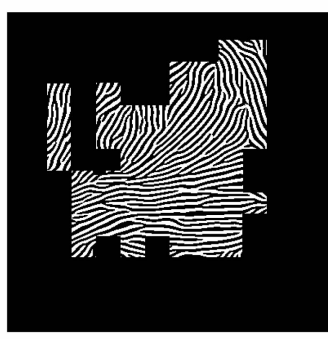

HMRF Result
Figure 8. Latent fingerprint sample from NIST 27 database.

\section{Conclusions and Future Work}

Modeling the enhancement process as hierarchical MRF on large patches as observation allows us to use a larger context for restoration, providing the ability to reason from available patches. Experimental results indicate that we are able to perform close to human assisted enhancement methods. The ability to reduce false minutiae detections in noisy images makes the approach useful for automatic processing in a variety of scenarios.

One of the primary disadvantages of the approach is that the time required for estimation of candidate patches from a large atlas is very large, making the enhancement process slow. We are currently working on indexing methods as well as parallel processing to improve the speed. Representation of fingerprints using the patch labels for matching is also extremely useful.

\section{References}

[1] A. M. Bazen and S. H. Gerez. Systematic methods for computation of directional fields and singular points of fingerprints. IEEE Trans. on PAMI, 24(7):905-919, 2002. 3

[2] S. Bernard, N. Boujemaa, D. Vitale, and C. Bricot. Fingerprint segmentation using the phase of multiscale gabor wavelets. In Proc. ACCV, Jan. 2002. 1

[3] R. Cappelli, A. Erol, D. Maio, and D. Maltoni. Synthetic fingerprint-image generation. In Proc. ICPR, pages 475478, Sept. 2000. 6

[4] R. Cappelli, D. Maio, and D. Maltoni. Semi-automatic enhancement of very low quality fingerprints. In Proc. ICPR, pages 678-683, Sept. 2009. 5, 6, 7

[5] R. Chellappa and A. K. Jain. Markov Random Fields: Theory and Applications. Academic Press, 1993. 4

[6] S. Chikkerur, V. GovindaRaju, and E. N. Cartwright. Fingerprint image enhancement using stft analyis. Proc. of ICAPR, 40(1):20-29, 2005. 1

[7] S. C. Dass. Markov random field models for directional field and singularity extraction in fingerprint images. IEEE Trans. on Image Processing, 3(1):1358-1367, 2004. 2, 3

[8] P. F. Felzenszwalb and D. P. Huttenlocher. Efficient belief propagation for early vision. International Journal of Computer Vision, 70(1):41-54, 2006. 4

[9] S. Greenberg, M. Aladjem, D. Kogan, and I. Dimitrov. Fingerprint image enhancement using filtering techniques. In Proc. ICPR, Sept. 2000. 1

[10] M. D. Gupta, S. Rajaram, N. Petrovic, and T. S. Huang. Models for patch based image restoration. In Proc. Computer Vision and Pattern Recognition Workshop, 2006. 2

[11] E. Henry. Classification and uses of Fingerprints. Routledge, London, 1900. 3

[12] L. Hong, Y. Wang, and A. K. Jain. Fingerprint image enhancement : Algorithm and performance evaluation. Trans. on PAMI, 21(4):777-789, 1998. 1, 5

[13] http://bias.csr.unibo.it/fvc2006. Fingerprint verification competition, 2006. 5

[14] H. W. Kuhn. The hungarian method for the assignment problem. Naval Research Logistics Quarterly, 2:83-97, 1955. 6

[15] K. C. Lee and S. Prabhakar. Probabilistic orientation field estimation for fingerprint enhancement and verification. In In proceedings of Biometric Symposium, Sept. 2008. 2

[16] L. Liu and T. Dai. A hybrid fingerprint enhancement algorithm. In Proc. IPCV'06, pages 35-40, Aug. 2006. 2

[17] D. Maio, D. Maltoni, A. K. Jain, and S. Prabhakar. Hand Book of Fingerprint Recognition. Springer-Verlag, 2003. 1

[18] L. O'Gormann and J. Nickerson. An approach to fingerprint filter design. Pattern Recognition, 22(1):29-38, 1989. 1

[19] B. G. Sherlock, D. M. Monro, and K. Millard. Fingerprint enhancement by directional fourier filtering. Visual Signal Image Processing, 141(6):87-94, 1994. 1

[20] B. W. Silverman. Density estimates for statistics and data analysis. In CRC Press, 1986. 3, 4

[21] J. Yang, L. Liu, T. Jiang, and Y. Fan. A modified gabor filter design method for fingerprint image enhancement. Pattern Recognition Letters, 24(1):1805-1817, 2003. 2 